\title{
A Call to Action by the Italian Mesotherapy Society on Scientific Research
}

\author{
Massimo Mammucari (iD) \\ Teresa Paolucci \\ Domenico Russo ${ }^{3}$ \\ Enrica Maggiori ${ }^{1}$ \\ Raffaele Di Marzo 4 \\ Alberto Migliore (iD ${ }^{5}$ \\ Umberto Massafra (iD ${ }^{5}$ \\ Gianpaolo Ronconi ${ }^{6}$ \\ Paola Emilia Ferrara ${ }^{6}$ \\ Fabio Gori ${ }^{7}$ \\ Barbara Bifarini $^{7}$ \\ Stefano Brauneis (iD ${ }^{8}$ \\ Renato Vellucci iD 9 \\ Rocco Domenico Mediati ${ }^{9}$ \\ Bartolomeo Violo 10 \\ Silvia Natoli iD 11 \\ Carmen Pediliggieri ${ }^{12}$ \\ Cristiana Di Campli ${ }^{12}$ \\ Maria Chiara Collina ${ }^{12}$
}

'Primary Care Unit, ASL RM I, Rome, Italy; ${ }^{2}$ Unit of Physical Medicine and Rehabilitation,

G. D' Annunzio University of Chieti-Pescara,

Department of Oral Medical Science and

Biotechnology (DSMOB), Chieti, Italy; 3"'San

Marco" Hospice and Palliative Care, Latina,

Italy; ${ }^{4}$ Member of the Italian Society of

Mesotherapy, Rome, Italy; ${ }^{5}$ Unit of

Rheumatology, San Pietro Fatebenefratelli

Hospital, Rome, Italy; ${ }^{6}$ Physical Medicine and

Rehabilitation Unit, IRCCS, Catholic University

of Sacred Heart, Rome, Italy; ${ }^{7}$ Section of

Anaesthesia, Intensive Care, and Pain Medicine,

University Hospital Santa Maria Della

Misericordia, Perugia, Italy; ${ }^{8}$ Pain Centre "Enzo

Borzomatl", University Hospital of Rome

"Policlinico Umberto I", Rome, Italy;

${ }^{9}$ Anaesthesiology Department, University

Hospital Careggi, Florence, Italy; ${ }^{10}$ Pain

Therapy Unit, S. Spirito Nuovo Regina

Margherita Hospital, Rome, Italy; " Department

of Clinical Science and Translational Medicine,

Tor Vergata University, Rome, Italy;

${ }^{12}$ Department of Vascular Surgery, Limb

Salvage and Diabetic Foot, IDI-IRCCS, Rome, Italy

Correspondence: Massimo Mammucari

Primary Care Unit, Local Health, ASL RM

I, Rome, 00165, Italy

Tel +39-06-6I560695

Email massimo.mammucari@libero.it

\begin{abstract}
Mesotherapy (local intradermal therapy, LIT) is a technique used to slowly spread drugs in tissues underlying the site of injection to prolong the pharmacological effect with respect to intramuscular injection. Recommendations for proper medical use of this technique have been made for pain medicine and rehabilitation, chronic venous disease, sport medicine, musculoskeletal disorders, several dermatological conditions, skin ageing, and immune-prophylaxis. Although mesotherapy is considered a valid technique, unresolved questions remain, which should be answered to standardize methodology and dosing regimen as well as to define the right indications in clinical practice. New randomized controlled trials are needed to test single products (dose, frequency of administration, efficacy and safety). Even infiltration of substances for dermo-cosmetic purposes must be guided by safety and efficacy tests before being proposed by mesotherapy. In this article, we put forth a preclinical and clinical research plan and a health technology assessment as a call to action by doctors, researchers and scientific societies to aid national health authorities in considering mesotherapy for prevention, treatment and rehabilitation paths.
\end{abstract}

Keywords: mesotherapy, intradermal therapy, research plan

\section{Introduction}

Mesotherapy (local intradermal therapy, LIT) is a technique used in individualized treatment paths in combination with other pharmacological or non-pharmacological therapies. The aim is to obtain benefits with lower drug doses when other options have failed or cannot be used, or when there are no other treatment options. ${ }^{1}$ This technique involves the infiltration of a small quantity of the drug into the superficial layer of the skin, in correspondence with the area of the pain or other clinical conditions. ${ }^{2}$ When injected intradermally (ID), the drug diffuses into tissues and underlying joints, remaining for a longer period of time than in the case of intramuscular administration. ${ }^{2-5}$

The recommendation of the Italian Society of Mesotherapy (SIM) is to inject only those drugs which have been chosen on the basis of diagnosis and authorized indications, avoiding the risk of mixing multiple products without proof of safety and efficacy. ${ }^{1-3}$ On the basis of this direction, LIT is used both to reduce localized pain and to manage patients with rehabilitation needs and sports injuries, especially when localized pain must be managed with the minimum effective drug dose. ${ }^{4,5}$ Mesotherapy has also been applied to other fields, such as management of some signs and symptoms of chronic venous disease ${ }^{6-8}$ and in some dermatological conditions. $^{9}$ In addition, LIT has been applied to manage some cases of skin 
ageing, such as wrinkling, loss of elasticity, laxity and rough-textured appearance. ${ }^{10}$ Finally, ID has been identified as a useful administration route in immune prophylaxis. $^{11-13}$

It should be noted, however, that in spite of its wide diffusion mesotherapy still presents many unresolved questions, such that further preclinical and clinical study is needed to define its role in clinical practice. ${ }^{14}$ In this context, researchers must first identify priority areas in which the therapy is most useful so as to concentrate research toward development and standardisation of mesotherapy technique in clinical practice. To this end, 21 experts of the Italian Society of Mesotherapy have drawn up a document to summarise available scientific evidence in favour of the use of mesotherapy in different fields of application. ${ }^{15}$ Based on this paper, we have extrapolated the main open questions and designed a preclinical and clinical research plan to appropriately apply mesotherapy technique in standard care pathways. Some studies are designed to confirm clinical results which are already available, while others are needed to investigate new areas of application. In addition to preclinical and clinical research, we have identified some areas of study for a health technology assessment (HTA).

\section{Pre-Clinical and Clinical Investigation Depth of Infiltration}

Although preclinical studies have reported slow and persistent diffusion in underlying tissues after ID, ${ }^{16-18}$ understanding how drugs reach underlying tissues and joints would be beneficial to researchers and practitioners. Binaglia et $\mathrm{al}^{16}$ have shown that an intradermally inoculated anaesthetic diffuses into underlying tissues while maintaining tissue concentrations longer than in the intramuscular route. The same result was obtained with the administration of ketoprofen. ${ }^{17}$ Pitzurra et al confirmed these results after intradermal inoculation of antibiotics. ${ }^{18}$ It is now believed that inoculation within a depth of $4 \mathrm{~mm}$ diffuses a drug more slowly than at a greater depth. ${ }^{1,4,5,15}$

No available data establish a dose-response curve for an intradermally or subcutaneously injected active substance. In this regard, we underline that evaluation of the role played by the absorption of the intradermally injected drug in the systemic circulation would clarify some of the unresolved questions that we have referred to. In fact, if the amount of systemically absorbed active ingredient plays an irrelevant role on the analgesic effect, ID could be re-evaluated, not only in terms of the dose-sparing effect but also for the lesser impairment of systemic organs, a factor that often limits the use of systemically administered anti-inflammatory drugs and painkillers. In this phase of research, we suggest studying the doseresponse curve of the intradermal route with respect to other routes of administration.

\section{Mesodermal Modulation}

At present, we know that structures that could actively participate in pain modulation are present in the dermis, ${ }^{1,19}$ such that a relationship between the analgesic effect induced by mesotherapy and the dermal cells cannot be excluded. Schwann cells intimately associated with unmyelinated nociceptive nerves, for example, are known to be mechanically sensitive and may transmit nociceptive information to the central nervous system. ${ }^{19}$ These mechanisms could explain some favourable clinical results which have been obtained with mesotherapy infiltration of centrally acting drugs. ${ }^{1,4}$ Thanks to drug-dermal cell interactions, mesodermal modulation could represent a new line of pharmacological research. For this reason, investigation of the relationships between dermal cells and drugs injected for analgesic (eg, opioids, anti-inflammatories, anaesthetics or muscle relaxants) or preventive purposes would be opportune.

\section{Dermal Pharmacokinetics}

The concentration of injected products represents another area of investigation necessary for understanding the dose of the drug to be injected on a given surface $\left(\right.$ dose $\left./ \mathrm{cm}^{2}\right)$. The role of superficial infiltration of inert substances should also be further investigated. ${ }^{20,21}$ Among the various fields of application of mesotherapy, immune prophylaxis represents a crucial area. ${ }^{13}$ In fact, it has been shown that skin can amplify the effects of an intradermally administered antigen: a smaller amount of substance can develop a better immune response. This dose-sparing effect could increase vaccine availability for worldwide immunization campaigns. Kenney et al have shown that ID of one fifth the standard intramuscular dose of an influenza vaccine elicited immunogenicity similar to or better than that elicited by intramuscular injection. ${ }^{11}$ These results suggest that ID could be used to expand supplies of vaccine, although further studies are needed before this strategy can be recommended for routine use. ${ }^{12,13}$ 
The dermis could represent the target of new therapies designed precisely to identify the minimum effective dose and reduce the risk of adverse events due to high doses of the drug. ${ }^{9}$ A line of research able to confirm this principle could have a broad clinical and economic impact in dermatology, oncology, pain medicine and other areas.

\section{Dermal Pharmacodynamics in Pain Management}

In past years, researchers suggested drug mixtures administered with empirically selected concentrations. ${ }^{22,23}$ The use of a single drug seems to reduce the risk of drug interactions and local side effects. ${ }^{1-4}$

Interestingly, studies that tested administration of both multiple active substances in the same syringe and a single analgesic drug produced the same significant clinical outcomes in patients with acute, subacute or chronic pain. ${ }^{1,4,5}$ It cannot therefore be excluded that the use of mixtures may overdose drugs. Furthermore, different efficacies have been reported among various analgesic drugs, ${ }^{24-27}$ a circumstance which is probably due to variations of pharmacological potencies or abilities to remain longer in underlying tissues.

The placebo effect induced by needles should also be studied to measure the analgesic role that micro-puncture could play. ${ }^{28}$ A comparison of two patient groups treated with anaesthetic-mesotherapy or needle puncture alone (dry mesotherapy) showed that both treatments induce pain control. ${ }^{27}$ Therefore, evaluation of the role played by each single drug with respect to a mixture or placebo (dry mesotherapy) would be useful, as would comparison of different therapeutic strategies (mesotherapy versus systemic therapy) in order to confirm interesting data showing a non-inferiority of mesotherapy with respect to the systemic route. ${ }^{29}$ Different subgroups of patients divided by age, sex, chronic diseases and comorbidities should be studied.

\section{Dermal Pharmacodynamics in the Management of Other Diseases}

Preliminary data indicate that mesotherapy allows for the management of signs and symptoms of chronic venous disease. ${ }^{7,8}$ Evaluating the effects of this administration route in patients with chronic vascular damage would be worthwhile, in addition to assessing standard procedures for the management of skin lesions (eg diabetic or therapy resistant patients with skin ulcers).
Local infiltrations are often used in many dermatological conditions. ${ }^{9}$ Nonetheless, it would be useful to understand the role of dermal infiltration versus intra lesion administration.

Some preliminary data indicate that mesotherapy technique could also be applied to oral mucosa. ${ }^{30}$ Applications in the dental field for both therapeutic and cosmetic purposes must be carefully tested before being proposed for routine clinical practice.

\section{Role of the Intradermal Route in the Management of Skin Imperfections}

Criticisms have recently been raised about the indiscriminate use of substances for aesthetic purposes injected into the dermis. ${ }^{31,32}$ The improper use of mesotherapy by unqualified personnel with mixtures of substances without a medical rationale has led to skin infections and many adverse reactions. ${ }^{33,34}$ For this reason, current guidelines regulate the application of mesotherapy in the treatment of skin ageing and other dermatological imperfections according to precise recommendations. ${ }^{15}$ Given the high number of receptors and skin cells, ${ }^{35}$ we believe it is necessary to design observational studies to investigate the long-term effects of the use of substances for aesthetic purposes. As many manufacturers market bio-revitalizing substances, it would be opportune to measure their effects compared to placebo (dry mesotherapy or sterile solution).

\section{Health Technology Assessment}

The economic impact of mesotherapy appears to be significant. ${ }^{36}$ Ad hoc studies should be designed to measure the impact of drug-sparing effects and avoided drug interactions on economic resources. Treatment algorithms that include mesotherapy could also be evaluated to assess the impact on individual patient care pathways. In fact, existing literature suggests that application of mesotherapy according to good clinical practice ${ }^{36}$ seems to be able to satisfy all stakeholders involved.

\section{Discussion}

Mesotherapy has proved to be no less effective than systemic therapy in the treatment of localized pain. ${ }^{37}$ Considering the usefulness of mesotherapy in clinical practice, it seems advisable to invest in research to identify those therapeutic pathways with the greatest saving of drugs. To this end, we have developed a series of open questions that require scientific confirmation (Figure 1). We have also designed a preclinical 


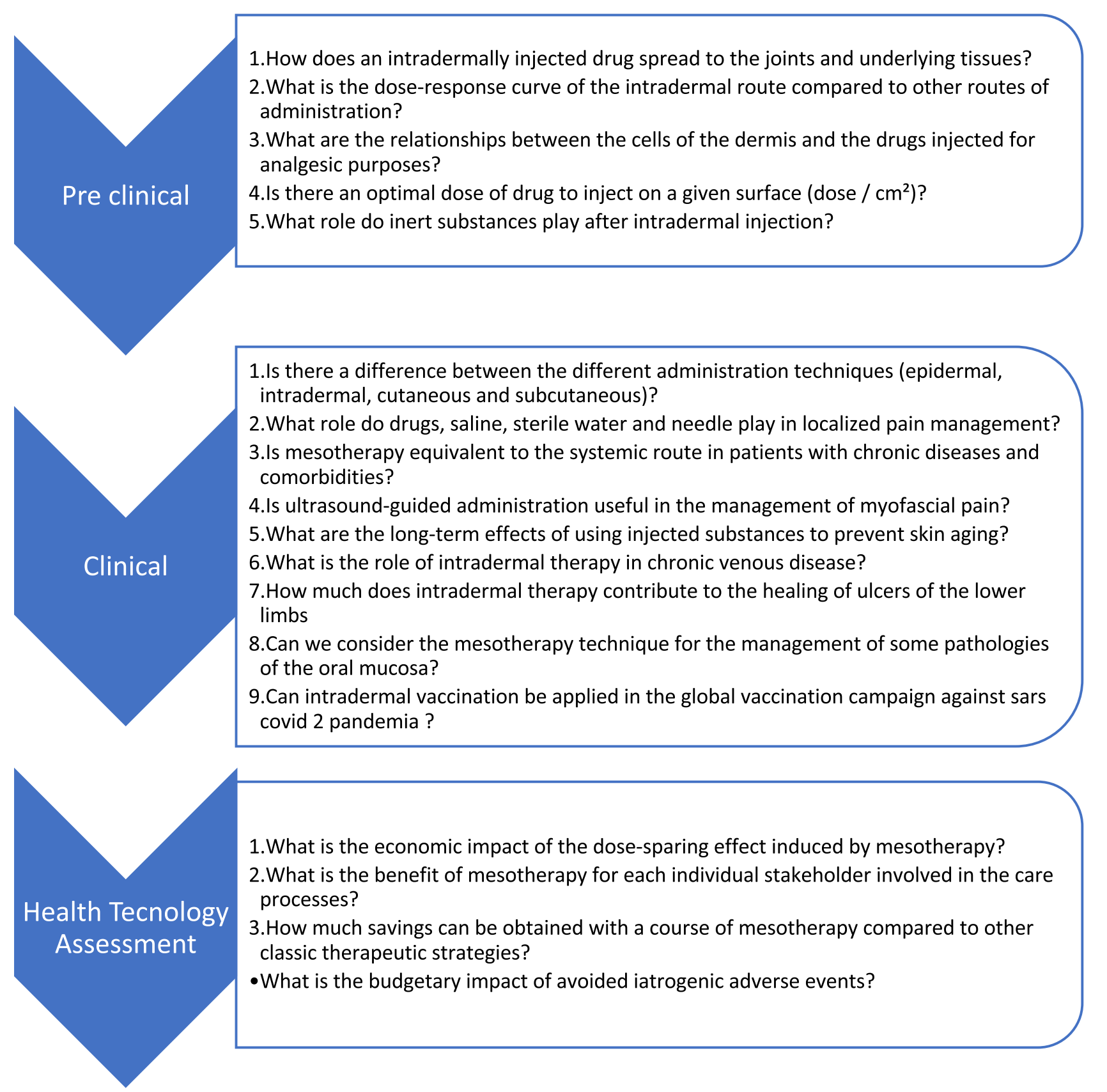

Figure I The main open questions on mesotherapy.

Note: Each question could be the subject of an ad hoc research plan.

and clinical research strategy that could be pursued to include mesotherapy technique in standard care pathways (Figure 2).

Localized treatment has several advantages, including a reduction in the dose of active ingredients, lower frequency of administration, efficacy comparable to the systemic route, lower risk of drug interactions with other systemic treatments, and clinical benefits, in particular in elderly subjects who take many drugs for various concomitant diseases. Standardizing this technique to introduce its reuse in care pathways could produce significant savings in resources. Therefore, solid evidence on efficacy and tolerability must be produced to include mesotherapy in standard care. To this end, we are calling on researchers to pursue this goal. Research could definitively establish the drug-sparing effect of the intradermal administration pathway with practical implications for the immunological field as well, by, for example, reducing the dose necessary to obtain immunization against COVID-19. ${ }^{13}$ In addition, research could help us 




Figure 2 Research pathway do develop mesotherapy.

Abbreviations: RWE, Real World Evidence; RWD, Real World Data; ID, intradermal; SC, subcutaneous.

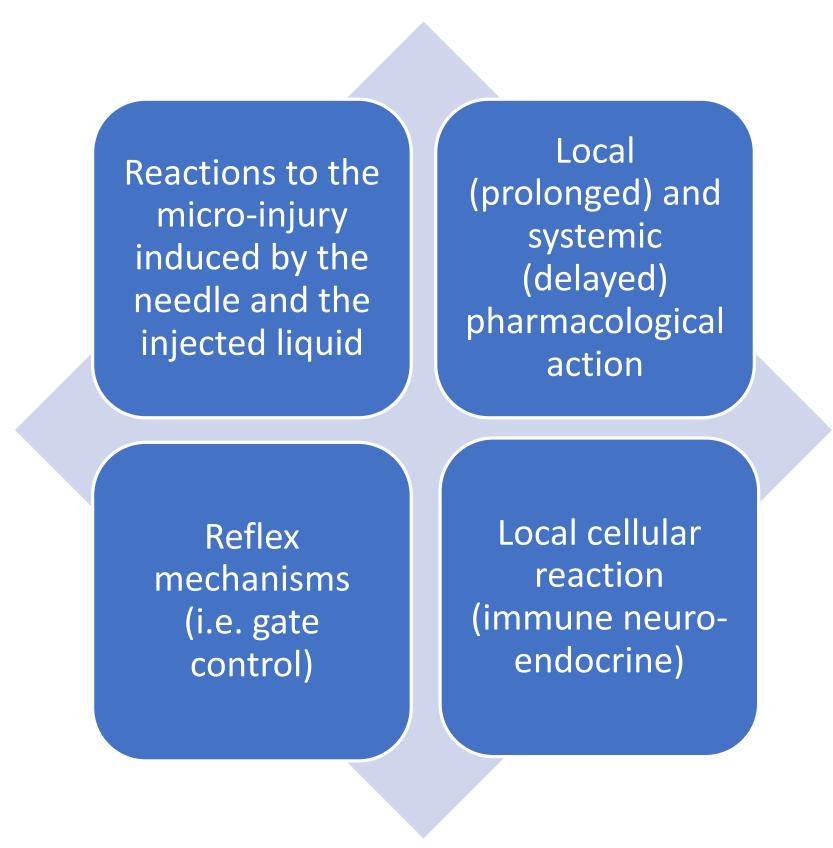

Figure 3 Possible mechanisms of action of the local intradermal therapy. Notes: The set of mechanisms induced by "mesotherapy" is called "mesodermal modulation". Data from Mammucari et al. ${ }^{15}$

to refine delivery techniques and to choose combination therapies, frequency and number of sessions.
Finally, it would also be important to understand the role of the dermis as a target organ for new therapies. In fact, ID seems to stimulate a series of skin reactions that directly or indirectly modulate a series of clinically significant responses (Figure 3).

\section{Conclusion}

Mesotherapy has reached a considerable degree of diffusion, and available data confirm its usefulness. The term mesotherapy has given way to the technical denomination of "local intradermal therapy". Today we have a new goal: to understand the role of the "mesodermal modulation" that the dermis implements in response to the inoculation of substances. It is an invitation to action by doctors and researchers to help health authorities to consider "mesotherapy" in the path of prevention, treatment and rehabilitation.

\section{Acknowledgment}

We thank Lexis for revising the English version of this article.

\section{Funding}

This article was not supported with funding. 


\section{Disclosure}

Prof. Dr. Silvia Natoli reports personal fees from Grunenthal Italia, grants from Grunenthal Italia, personal fees from Mylan, personal fees from Angelini, personal fees from Sandoz, outside the submitted work. All the other authors declare no conflicts of interest.

\section{References}

1. Mammucari M, Maggiori E, Russo D, et al. Mesotherapy: from historical notes to scientific evidence and future prospects. Sci World J. 2020. doi:10.1155/2020/3542848

2. Mammucari M, Gatti A, Maggiori S, et al. Mesotherapy, definition, rationale and clinical role: a consensus report from the Italian Society of Mesotherapy. Eur Rev Med Pharmacol Sci. 2011;15:682-694.

3. Mammucari M, Vellucci R, Mediati RD, et al. What is mesotherapy? Recommendations from an international consensus? Trends Med. 2014;14:1-10

4. Mammucari M, Gatti A, Maggiori S, et al. Role of mesotherapy in musculoskeletal pain: opinions from the italian society of mesotherapy. Evid Based Complement Alternat Med. 2012;2012:436959. doi:10.1155/2012/436959

5. Paolucci T, Bellomo RG, Centra MA, et al. Mesotherapy in the treatment of musculoskeletal pain in rehabilitation: the state of the art. J Pain Res. 2019;30(12):2391-2401. doi:10.2147/JPR.S209610

6. Arosio E, Ferrari G, Santoro I, et al. A placebo-controlled, double blind study of mesoglycan in the treatment of chronic venous ulcers. Eur J Endovasc Surg. 2001;22:365-372. doi:10.1053/ejvs.2001.1478

7. Maggiori E, Bartoletti CA, Maggiori S, et al. Local intradermotherapy (ITD) with mesoglicano in PEFS and IVLC, retrospective study. Trends Med. 2010;10(1):73-78.

8. Maggiori E, Bartoletti E, Mammucari M. Intradermal therapy (mesotherapy) with lymdiaral in chronic venous insufficiency and associated fibrosclerotic edema damage: a pilot study. $J$ Altern Complement Med. 2013;19(9):777-781. doi:10.1089/acm.2012.0734

9. Canzona F, Mammucari M, Tuzi A, et al. Intradermal Therapy (mesotherapy) in Dermatology. J Dermatol \& Skin Sci. 2020;2 (1):22-25.

10. El-Domyati M, El-Ammawi TS, Moawad O, et al. Efficacy of mesotherapy in facial rejuvenation: a histological and immunohistochemical evaluation. Int $J$ Dermatol. 2012;51(8):913-919. doi:10.1111/j.1365-4632.2011.05184.x

11. Kenney RT, Frech SA, Muenz LR, et al. Dose sparing with intradermal injection of influenza vaccine. $N$ Engl J Med. 2004;351 (22):2295-2301. doi:10.1056/NEJMoa043540

12. Nicolas JF, Guy B. Intradermal, epidermal and transcutaneous vaccination: from immunology to clinical practice. Expert Rev Vaccines. 2008;7(8):1201-1214. doi:10.1586/14760584.7.8.1201

13. Migliore A, Gigliucci G, Di Marzo R, et al. Intradermal Vaccination: a Potential Tool in the Battle Against the COVID-19 Pandemic? Risk Manag Healthc Policy. 2021;14:2079-2087. doi:10.2147/RMHP. S309707

14. Mammucari M, Maggiori E, Antonaci L, et al. Intradermal therapy recommendations for standardization in localized pain management by the Italian Society of Mesotherapy. Minerva Med. 2019.

15. Mammucari M, Russo D, Maggiori E, et al. Evidence based recommendations on mesotherapy: an update from the Italian society of Mesotherapy. Clin Ter. 2021;171(1):e37-e45. doi:10.7417/ CT.2021.2278

16. Binaglia L, Maconi P, Pitzurra M. The diffusion of intradermally administered procaine. J Mesother. 1981;1:15-28.

17. Binaglia L, Maconi P, Pitzurra M. Absorption of Na ketoprofen administered intradermally. J Mesother. 1981;1:85-91.
18. Pitzurra M, Cavallo R, Farinelli S, et al. The intradermal inoculation of antibiotics: some experimental data. J Mesother. 1982;1:9-14.

19. Abdo H, Calvo-Enrique L, Lopez JM, et al. Specialized cutaneous Schwann cells initiate pain sensation. Science. 2019;365 (6454):695-69922. doi:10.1126/science.aax6452

20. Ferrara PE, Ronconi G, Viscito R, et al. Efficacy of mesotherapy using drugs versus normal saline solution in chronic spinal pain: a retrospective study. Int $J$ Rehabil Res. 2017;40(2):171-174. doi:10.1097/MRR.0000000000000214

21. Ronconi G, Ferriero G, Nigito C, et al. Efficacy of intradermal administration of diclofenac for the treatment of non specific chronic low back pain: result from a retrospective observational study. Eur J Phys Rehabil Med. 2019;54-55.

22. Pistor M. Qu'est-ce que la mésothérapie? Chir Dent Fr. 1976;46 (288):59-60.

23. Vedamurthy M. Mesotherapy. Indian J Dermatol Venereol Leprol. 2007;73(1):60-62. doi:10.4103/0378-6323.30661

24. Costantino C, Marangio E, Coruzzi G. Mesotherapy versus systemic therapy in the treatment of acute low back pain: a randomized trial. Evid Based Complement Alternat Med. 2011;317183. doi:10.1155/ 2011/317183

25. Chen L, Li D, Zhong J, et al. Therapeutic effectiveness and safety of mesotherapy in patients with osteoarthritis of the knee. EvidenceBased Compl Altern Med. 2018;4:6513049.

26. Ferrara PE, Nigito C, Maccauro G, et al. Efficacy of diclofenac mesotherapy for the treatment of chronic neck pain in spondylartrosis. Minerva Med. 2018;110-113.

27. Paolucci T, Piccinini G, Dan Trifan P, et al. Efficacy of trigger points mesotherapy for the treatment of chronic neck pain: a short-term retrospective study. Int J Phy Ther Rehabil. 2016;2:113.

28. Crema P, Mancia M. Reflex action in mesotherapy. $J$ Mesother. 1981;1(1):29-40.

29. Kocak AO. Intradermal mesotherapy versus systemic therapy in the treatment of musculoskeletal pain: a prospective randomized study. Am J Emerg Med. 2019;37(11):2061-2065. doi:10.1016/j. ajem.2019.02.042

30. Yussif NMA, El-Saadany BAE. Oral Mesotherapy. Rep Opinion. 2016;8(7):7-12.

31. Atiyeh BS, Ibrahim AE, Dibo SA. Cosmetic mesotherapy: between scientific evidence, science fiction, and lucrative business. Aesthetic Plast Surg. 2008;32(6):842-849. doi:10.1007/s00266-008-9195-x

32. Sivagnanam G. Mesotherapy - The french connection. J Pharmacol Pharmacother. 2010;1(1):4-8. doi:10.4103/0976-500X.64529

33. Veraldi S, Spigariolo CB, Cusini M, et al. Skin infections by Mycobacterium chelonae following mesotherapy: a report of two cases and review of the literature [published online ahead of print, 2020 Apr 15]. J Cosmet Dermatol. 2020. doi:10.1111/jocd.13441

34. Carbonne A, Brossier F, Arnaud I, et al. Outbreak of nontuberculous mycobacterial subcutaneous infections related to multiple mesotherapy injections. J Clin Microbiol. 2009;47(6):1961-1964. doi:10.1128/ JCM.00196-09

35. Zhang S, Duan E. Fighting against Skin Aging: the Way from Bench to Bedside. Cell Transplant. 2018;27(5):729-738. doi:10.1177/ 0963689717725755

36. Basile M, Rumi F, Palmeri M, et al. Cost-of-illness dell'osteoartrite in Italia: burden economico dell'inappropriatezza prescrittiva. Glob Reg Health Technol Assess. 2020;7(1):101-108. doi:10.33393/ grhta.2020.2130

37. Faetani L, Ghizzoni D, Ammendolia A, et al. Safety and efficacy of mesotherapy in musculoskeletal disorders: a systematic review of randomized controlled trials with meta-analysis. J Rehabil Med. 2021;53(4):jrm00182. doi:10.2340/16501977-2817 
Video abstract

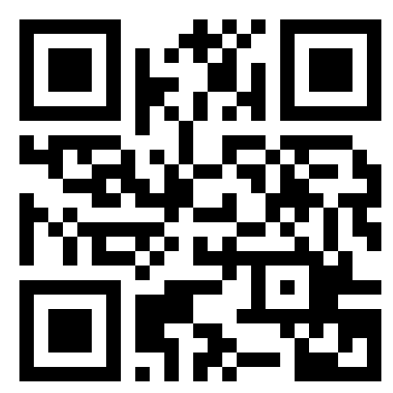

Point your SmartPhone at the code above. If you have a QR code reader the video abstract will appear. Or use: https://youtu.be/bluRhGQsUjs

\section{Publish your work in this journal}

Drug Design, Development and Therapy is an international, peerreviewed open-access journal that spans the spectrum of drug design and development through to clinical applications. Clinical outcomes, patient safety, and programs for the development and effective, safe, and sustained use of medicines are a feature of the journal, which has also been accepted for indexing on PubMed Central. The manuscript management system is completely online and includes a very quick and fair peer-review system, which is all easy to use. Visit http://www. dovepress.com/testimonials.php to read real quotes from published authors. 